Asian-Australasian Journal of

Food Safety and Security

ISSN 2523-1073 (Print) 2523-2983(Online)

https://www.ebupress.com/journal/aajfss/

\title{
Article \\ Judicious and non-judicious use of colistin sulfate in indoor poultry experimentation and its effect on haematological parameters and body weight in broiler
}

Saifa Nasar Trisha, Md. Shafiqul Islam*, Md. Rakibul Hasan, Md. Mahmudul Hasan Sikder and Most. Shumi Akhter Sathi

Department of Pharmacology, Faculty of Veterinary Science, Bangladesh Agricultural University, Mymensingh-2202, Bangladesh

*Corresponding author: Professor Dr. Md. Shafiqul Islam, Department of Pharmacology, Bangladesh Agricultural University, Mymensingh, Bangladesh. E-mail: shafiqpharma@yahoo.co.uk

Received: 24 August 2021/Accepted: 18 November 2021/ Published: 30 November 2021

Copyright (C) 2021 Saifa Nasar Trisha et al. This is an open access article distributed under the Creative Commons Attribution 4.0 International License (https://creativecommons.org/licenses/by/4.0/), which permits unrestricted use, distribution, and reproduction in any medium, provided the original work is properly cited.

Abstract: The aim of this study was to evaluate the effects of discriminate and indiscriminate use of colistin sulfate on body weight and haematological parameters in broiler. Day-old-broiler chicks were collected and reared for up to 31 days. The chicks were randomly divided into control, discriminate and indiscriminate antibiotic group. The discriminate group was treated with colistin sulfate antibiotic (Eskolis-24) for one week (from 16th to 22th day) followed by one week withdrawal period. In case of indiscriminate group, the poultry were treated with colistin sulfate antibiotic for 14 days (from 16th to 29th day) without any withdrawal period until sacrifice. The body weight of the birds were recorded daily. The mean body weight was highest in indiscriminate group $(1408.22 \pm 133.49 \mathrm{gm})$ followed by discriminate group $(1330.15 \pm 134.93 \mathrm{gm})$ and control group $(1243.81 \pm 173.19 \mathrm{gm})$. The differences among means of three groups were not statistically significant. The Total erythrocyte count (Million/mm3) of control, discriminate, and indiscriminate groups were $2.53 \pm 0.05$, $2.56 \pm 0.08$, and $2.52 \pm 0.08$ respectively. The hemoglobin ( $\mathrm{gm} \%$ ) were $7.06 \pm 0.09,7.03 \pm 0.12$, and $7.11 \pm 0.09$ respectively and the Packed Cell Volume (\%) were 19.66 $\pm 1.11,19.33 \pm 0.95$, and $20.16 \pm 0.70$ respectively. The results found were not statistically significant among the groups for TEC, Hb and PCV respectively. Total lymphocyte $(\%)$ count were $67.16 \pm 0.60,70 \pm 1.15$, and $71.83 \pm 1.30$ respectively. Total neutrophil (\%) count were $32.83 \pm 0.60,28.83 \pm 1.42$, and $26.83 \pm 1.10$ respectively. Total eosinophil $(\%)$ count were $0 \pm 0,1 \pm 0.44$, and $1.16 \pm 0.40$ respectively. Total basophil $(\%)$ count were zero $(0)$ and total monocyte $(\%)$ count were $0 \pm 0$, $0.17 \pm 0.17$, and $0.17 \pm 0.17$ respectively. In our experiment, both discriminate and indiscriminate groups showed no significant differences of lymphocyte, neutrophil, eosinophil, basophil and monocyte. Therefore, discriminate and indiscriminate use of colistin sulfate for two weeks has no effect on haematological parameters of broiler poultry. Further experiment needed for more concise conclusion.

Keywords: colistin sulphate; broiler; hematological parameter; body weight

\section{Introduction}

Bangladesh is an agricultural country and livestock sector is one of the most sustainable sub-sector of our economy. Contribution of livestock sector to GDP is $1.47 \%$. Therefore it is necessary to provide long term food security by ensuring a sustainable agricultural system. It is therefore important to have a profitable, sustainable and environment-friendly agricultural system in order to ensure long-term food security for people. Livestock is 
the most viable sector in the economy of the four sub-sectors of agriculture (crop, livestock, fisheries and forestry). At present livestock contributes 1.47\% to GDP (Constant Prices) on the basis of value added through its production of milk, eggs, meat and hides and skins (Hossan and Salim, 2018).

Currently, approximately $80 \%$ of all food producing animals and birds receive medication for part or most of their lives (Lee et al., 2001). Though factors related to farm management help to minimize the use of antibiotics, however, even if well managed, the increased density of livestock or poultry in intensive rearing operations requires an aggressive approach to disease control, which can lead to heavy prophylactic and therapeutic antibiotic use (Tollefson and Miller, 2011). Although these usages have value in terms of more production, unfortunately edible poultry tissues are being contaminated with harmful concentration of drug residues (Donoghue, 1993). In true sense, indiscriminate and injudicious use of drugs make poultry meat unsafe for human consumption if withdrawal period is not maintained accordingly (Lee et al., 2001). The use of antibiotics in food producing animals may result in the presence of residues in foodstuffs of animal origin. Protection of public health against possible harmful effects of veterinary drug residues is a relatively recent preoccupation. For prevention of diseases, enhancement of growth and feed efficacy, the use of antibiotic is increasing day by day (Singh et al., 2014) and antibiotics are used indiscriminate in poultry industries.

Colistin is an important antibiotic belonging to polymyxins group widely used in poultry industry for the treatment of collibacillosis, salmonellosis and clostridial diseases etc. through drinking water, as feed additive and through parenteral routes. Colistin is absorbed from gastrointestinal tract very slowly, therefore no detectable concentrations are found in plasma at ordinary doses. Colistin is a multicomponent antibiotic (polymyxins E) that is produced by strains of Bacillus polymyxa var. colistinus and is bactericidal to gramnegative bacteria, with a detergent-like mechanism. (Elverdam et al., 1981).

The two salt forms of polymyxin $\mathrm{E}$ (colistin) are widely available in human medicine, namely colistin sulfate an d colistimethate sodium (Bergen et al., 2006). The two major components, colistin A and B, differ only in the fatty acid side chain. Different pharmaceutical preparations of colistin may differ in the ratios of the major components (Decolin et al., 1997). There are two forms of colistin available for clinical use: colistin sulfate (for topical use) and colistin methanesulfonate (for parenteral and aerosol therapy). The use of colistin is limited by its potential nephrotoxicity, neurotoxicity, and hypersensitivity (Koch-Weser et al., 1970, Wolinsky et al., 1962). In the poultry production, colistin sulfate is indicated for the prevention and control of gastrointestinal diseases caused by Gram negative bacteria, as is the case of Escherichia coli and Salmonella spp. These last two bacteria cause diseases of great importance in the livestock industry (Mejia, 2003; Bozorgmehri, 2004; FAO, 2006; Gilbert, 2010; Collell and Segura, 2013). Colistin is used in aquaculture for the prevention of Gramnegative infections (Xu et al., 2012).

The parenteral administration of colistin needs extra care because they have narrow safety margin and overdoses leads to neurotoxicity and nephrotoxicity within no time. This toxicity is more in patients with renal insufficiency as colistin and other polymyxins are excreted primarily through kidneys. In rats (Wallace et al. 2008) reported severe nephrotoxicity with intravenous administration of colistin metahe-sulfonate (CMS). The colistin excreted unaltered through oral route in the faeces of food animals and it led to toxicity in nitrifying bacteria in the soil (Bressan et al., 2013). Therefore, indirectly it also effects the environment.

The indiscriminate use of such antimicrobials in livestock and poultry production is likely to accelerate the development of antibiotic resistance in pathogens, as well as in commensal organisms. This would result in treatment failures, economic losses and could act as source of gene pool for transmission to humans. Antibiotic treatment is also correlated with toxicity on the process of haematopoiesis which lead to changes in the blood parameters of the poultry (Stolker and Brinkman, 2005). Some antibiotics have effects on various blood components such as thrombocytopenia, anaemia, leukopenia, and so on (Al-Mayah and Al-Ahmed, 2005).

Hematological constituents usually reflect the physiological responsiveness of the animal to its external and internal environments and this is serving as a veritable tool for monitoring animal health. Hematological profile in animals is an important indicator of physiological or pathophysiological status of the body (Khan and Zafar, 2005). The changes found in the hematological profile may represent a possible warning sign of overdose in live animals, signs that can be detected in routine hematological investigations (Chung and wood, 1970). Besides, there is a lack study regarding the effects of colistin sulphate on blood profile of broiler chicken in Bangladesh.

\section{Materials and Methods}

\subsection{Ethical approval of laboratory animals}

The experimental broilers were used ethically and at the end of the experiment sacrificed humanely following the ethical and welfare guidelines set by the Animal Welfare and Experimental Ethics Committee of Bangladesh Agricultural University. 


\subsection{Experimental design}

18 day-old chicks (Cobb-500) were collected from the CP Bangladesh Company Ltd, Mymensingh. The birds were apparently healthy and were already vaccinated from the hatchery. The chicks were brooded firstly at $35^{\circ} \mathrm{C}$ temperature in the first week under intensive care and the temperature was successively decreased to $2.5^{\circ} \mathrm{C}$ per week till 30 days. The chicks were supplied with vitamin $\mathrm{C}$ (Rena C) And glucose with drinking water immediately after the transportation to remove stress. After 1-hour fresh starter feed was given to the chicks. The feed was purchased from the authorized dealer. On day 14th, all the birds were divided into three groups namely Group A (Control group), Group B (Discriminate group) and Group C (Indiscriminate group). Control group was supplied with normal drinking water whereas discriminate group was supplied with colistin sulphate in their drinking water at $2 \mathrm{gm} / \mathrm{liter}$ daily and indiscriminate group was supplied with colistin sulfate antibiotic $@ 10 \mathrm{gm} /$ litre drinking water. After 7 days antibiotic supply was stopped in discriminate group following withdrawal period, but continued in indiscriminate group until sacrifice.

\subsection{Weight measurement}

The birds were weighed from the first day to day 16 and recorded in a data book. The average body weights of the birds were taken into consideration. After the day 16 the birds of group A, B and C were weighed particularly and the results were recorded.

\subsection{Hematological analysis}

To perform the hematological examination the blood samples of group A, B, and C birds were collected and preserved separately.

\subsubsection{Determination of total erythrocyte count (TEC)}

Total erythrocyte count was done following the method described by (Lamberg and Rothstein., 1977). The wellmixed blood sample was drawn with red blood cell diluting pipette exactly up to 0.5 marks of the pipette. Outside of the tip of the pipette was wiped with cotton. Then the pipette was immediately filled with the red cell diluting fluid (Hayem's solution) up to 101 marks. The free end of the pipette was wrapped around with the rubber tube stretching to both the ends and held with the thumb and middle finger. The content of the pipette was mixed thoroughly by shaking with an 8-knot motion for 3-5 minutes. Then the counting chamber was placed with a special cover glass under a microscope using low power (10x) objectives. After discarding 2 or 3 drops of fluid from the pipette, a small drop was placed to the edge of the cover glass on the counting chamber as the entire area under the cover glass was filled by the fluid. One-minute time was spared to allow the cells to settle on the chamber under the cover glass. Taking 5 larger squares (4 in the 4 corners and the central one) of the central large square, the cells were counted from all the 80 small squares $(16 \times 5)$ under high power objectives (40x). After completion of counting, the total number of RBC was 35 calculated as a number of cells counted $\mathrm{x} 10,000$ and the result was expressed in million/ $\mu \mathrm{l}$ of blood.

\subsubsection{Determination of hemoglobin concentrations $(\mathrm{Hb} \%)$}

The N/10 hydrochloric acid (HCL) was taken in a graduated tube up to 2 marks with the help of a dropper. The well-homogenized blood sample was then drawn into the Sahli pipette up to $20 \mathrm{~cm}$. mark. The tip of the pipette was wiped with sterile cotton and the blood of the pipette was immediately transferred into the graduated tube containing hydrochloric acid. This blood and acid were thoroughly mixed by stirring with a glass stirrer. There was a formation of acid hematin mixture in the tube by haemolysing red blood cells by the action of HCL. The tube containing acid hematin mixture was kept standing in the comparator for 5 minutes. After that distilled water was added drop by drop. The solution was mixed well with a glass stirrer until the colour of the mixture resembled the standard colour of the comparator. The result was read in daylight by observing the height of the liquid in the tube considering the lower meniscus of the liquid column. The result was then expressed in $\mathrm{g} \%$. The above procedure was matched by the Helligehemometer method as described by (Lamberg and Rothstein., 1977).

\subsubsection{Determination of packed cell volume (PCV)}

The citrated well-mixed blood sample was drawn into special loading pipette (Wintrobe pipette). The tip of the pipette was inserted up to the bottom of a clean, dry Wintrobe hematocrit tube. Then the Wintrobe tube was filled from the bottom by pressing the rubber bulb of the pipette. As blood came out, the pipette was slowly withdrawn but the pressure was continued on the rubber bulb of the pipette so as to exclude air bubbles. The tip of the pipette was tried to keep under the rising column of blood to avoid foaming and the tube was filled 
exactly to the $10 \mathrm{~cm}$ mark. Then the Wintrobe hematocrit tube was placed in the centrifuge machine and was centrifuged for 30 minutes at $3000 \mathrm{rpm}$. Then, the hematocrit or PCV was recorded by reading the graduation mark; the percent volume occupied by the hematocrit was calculated by using the following formula as described by (Lamberg and Rothstein., 1977).

$\mathrm{PCV} \%=($ Height of the red cell volume in $\mathrm{cm} /$ Height of total blood in $\mathrm{cm}) \times 100$

\subsubsection{Determination of different leucocytes count (DLC)}

\subsubsection{Preparation of blood smear}

Two clean, dry, oil free glass slides were taken. One was placed horizontally on the table. A small medium drop of blood was placed on the right side of horizontal slide. A thin smear of blood was produced using the other slide having smooth unbroken end. The blood film was allowed to dry in the air.

\subsubsection{Staining of blood smear}

The smeared slide was places on the staining rack. The smear was flooded with Wright's stain and care was taken so that it does not fall. It was then left for 3 minutes for proper staining of the cells. Distilled water was added with the stain and was mixed by blowing using pipette. A bronze colour scum will appear at the top. The slide was then washed in running tape water. It was then allowed to dry in the air.

\subsubsection{Counting of different leucocytes}

The slide was placed under microscope. A fine distribution of cells was detected using 10X objective. A small drop of immersion oil was placed on the said focus. Different leucocytes were detected and counted using 100X objective. A parallel strip method of counting was followed to avoid duplication of counting. A total of 100 different leucocyte were counted. The cells counted were expressed in percentage.

\section{Results}

\subsection{Effects of colistin sulphate on body weight gain}

The average body weight gain was the highest in indiscriminate antibiotic group which was $1580.5 \pm 11.3 \mathrm{gm}$ whereas, the lowest was 1450.7 $\pm 9.9 \mathrm{gm}$ in control group represented in Figure 3. Discriminate antibiotic group showed moderate weight gain that was $1542 \pm 10.7 \mathrm{gm}$. The differences among means of three groups were not statistically significant. The multiple comparisons during one-way ANOVA revealed that there was no significant difference in mean between discriminate and indiscriminate antibiotic groups. The other two groups (Control and discriminate; Control and indiscriminate) showed no significant difference among means. In Figure 1, $2 \& 3$ represent, the average body weight gains of broilers of control, discriminate and indiscriminate antibiotic groups. Data are shown as Mean \pm Standard Error of Mean ( $n=6$ samples per group). The differences among means of three groups were not statistically significant.

\subsection{Effects of discriminate and indiscriminate use of colistin sulphate on haematological parameters in broiler poultry.}

\subsubsection{Total Erythrocyte Count (Million/ $\mathbf{m m}^{3}$ )}

The highest mean TEC was obtained from discriminate group that was $2.56 \pm 0.08$. The mean TEC of control and indiscriminate group were $2.53 \pm 0.05$ and $2.52 \pm 0.08$ respectively. The ordinary one-way ANOVA showed that the differences among means of control versus discriminate and indiscriminate groups were not statistically significant. There was no significant difference between discriminate and indiscriminate groups were represented in Figure 4.

In Figure 4 represents, effects of discriminate and indiscriminate use of colistin sulphate on total erythrocyte count (Million $\left./ \mathrm{mm}^{3}\right)$. Data are shown as Mean \pm Standard Error of Mean $(\mathrm{n}=6$ samples per group). The difference of means between control and discriminate; control and indiscriminate were not statistically significant. There was no significant difference between discriminate and indiscriminate groups.

\subsubsection{Haemoglobin (gm \%) count of three different groups}

The highest mean $\mathrm{Hb}$ was obtained from indiscriminate control group that was 7.11 \pm 0.09 . The mean $\mathrm{Hb}$ of control and discriminate group were $7.06 \pm 0.09$ and $7.03 \pm 0.12$ respectively. The ordinary one-way ANOVA showed that the differences among means of control versus discriminate and indiscriminate groups were not statistically significant. There was no significant difference between discriminate and indiscriminate groups and were represented in Figure 5. 
In Figure 5 represents, effects of discriminate and indiscriminate use of colistin sulphate on haemoglobin (gm \%). Data are shown as Mean \pm Standard Error of Mean ( $n=6$ samples per group). The difference of means between control and discriminate; control and indiscriminate were not statistically significant. There was no significant difference between discriminate and indiscriminate groups.

\subsubsection{Packed cell volume (\%) estimation}

The highest mean PCV was obtained from control group that was 19.66 \pm 1.11 . The mean PCV of discriminate and indiscriminate groups were $19.33 \pm 0.95$ and $20.16 \pm 0.70$ respectively. Packed cell volume (PCV) among the three different groups (Control, Discriminate and Indiscriminate) did not show any significant difference represented in Figure 6.

In Figure 6 represents, effects of discriminate and indiscriminate use of colistin sulphate on packed cell volume (\%). Data are shown as Mean \pm Standard Error of Mean ( $n=6$ samples per group). The differences of means between control and discriminate; control and indiscriminate were not statistically significant. There was no significant difference between discriminate and indiscriminate groups.

\subsubsection{Different Leucocyte Count (DLC) of three different groups \\ 3.2.4.1. Total lymphocyte $(\%)$ count}

The highest mean lymphocyte was obtained from indiscriminate group that was $71.83 \pm 1.30$. The mean lymphocyte of control and discriminate groups were $67.16 \pm 0.6$ and $70 \pm 1.15$ respectively. The ordinary one-way ANOVA showed that the differences among means of control versus discriminate and indiscriminate groups were statistically significant $(\mathrm{P}<0.05)$. There was significant difference between discriminate and indiscriminate groups were represented in Figure 7.

In Figure 7 represents, effects of discriminate and indiscriminate use of colistin sulphate on lymphocyte (\%). Data are shown as Mean \pm Standard Error of Mean ( $n=6$ samples per group). The difference of means between control and discriminate; control and indiscriminate were statistically significant $(P<0.05)$.

\subsubsection{Total neutrophil (\%) count of three different groups}

The highest mean neutrophil was obtained from control group that was $32.83 \pm 0.60$. The mean neutrophil of discriminate and indiscriminate groups were $28.83 \pm 1.42$ and $26.83 \pm 1.10$ respectively. The ordinary one-way ANOVA showed that the differences among means of control versus discriminate and indiscriminate groups were statistically significant $(P<0.05)$. There was significant difference between discriminate and indiscriminate groups were represented in 8 .

In Figure 8 represents, effects of discriminate and indiscriminate use of colistin sulphate on neutrophil (\%). Data are shown as Mean \pm Standard Error of Mean ( $\mathrm{n}=6$ samples per group). The difference of means between control and discriminate; control and indiscriminate were statistically significant $(P<0.05)$.

\subsubsection{Total eosinophil (\%) count of three different groups}

The highest mean eosinophil was obtained from indiscriminate group that was $1.16 \pm 0.40$. The mean eosinophil of control and discriminate groups were $0 \pm 0$ and $1 \pm 0.44$ respectively. Eosinophil among the three different groups (Control, Discriminate and Indiscriminate) did not show any significant difference represented in Figure 9.

In Figure 9 represents, effects of discriminate and indiscriminate use of colistin sulphate on eosinophil (\%). Data are shown as Mean \pm Standard Error of Mean ( $n=6$ samples per group). There was no significant difference between discriminate and indiscriminate groups.

\subsubsection{Total basophil (\%) count of three different groups}

The mean basophil was obtained among the three different groups (Control, Discriminate and Indiscriminate) were zero and did not show any significant difference represented in Figure 10.

\subsubsection{Total monocyte (\%) count of three different groups}

The mean monocyte was obtained from indiscriminate group that was $0.17 \pm 0.17$. The mean monocyte of control and discriminate group were $0 \pm 0$ and $0.17 \pm 0.17$ respectively. Monocyte among the three different groups (Control, Discriminate and Indiscriminate) did not show any significant difference represented in Figure 11. In Figure 11 represents, effects of discriminate and indiscriminate use of colistin sulphate on monocyte (\%). Data are shown as Mean \pm Standard Error of Mean ( $n=6$ samples per group). There was no significant difference between discriminate and indiscriminate groups. 


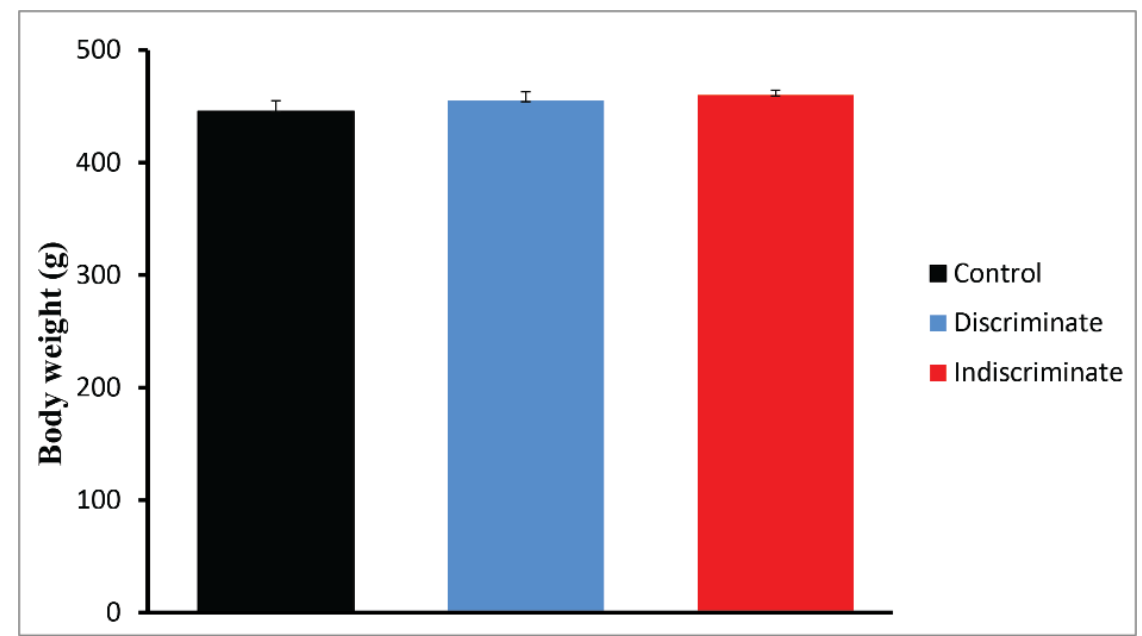

Figure 1. Body weight (gm) on $16^{\text {th }}$ day.

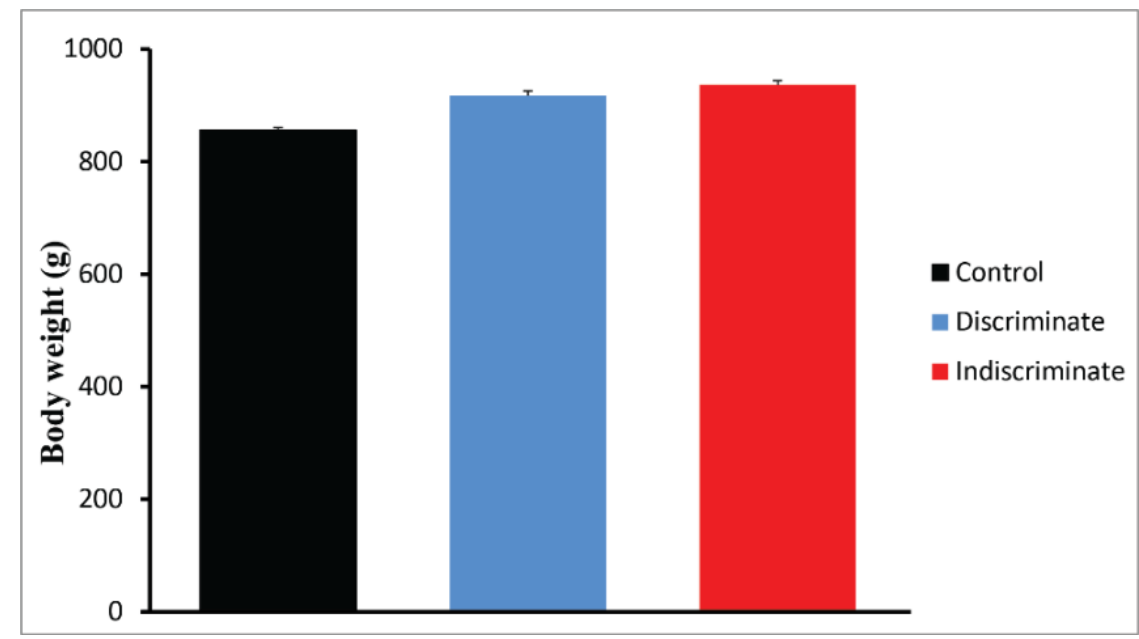

Figure 2. Body weight (gm) on $23^{\text {rd }}$ day.

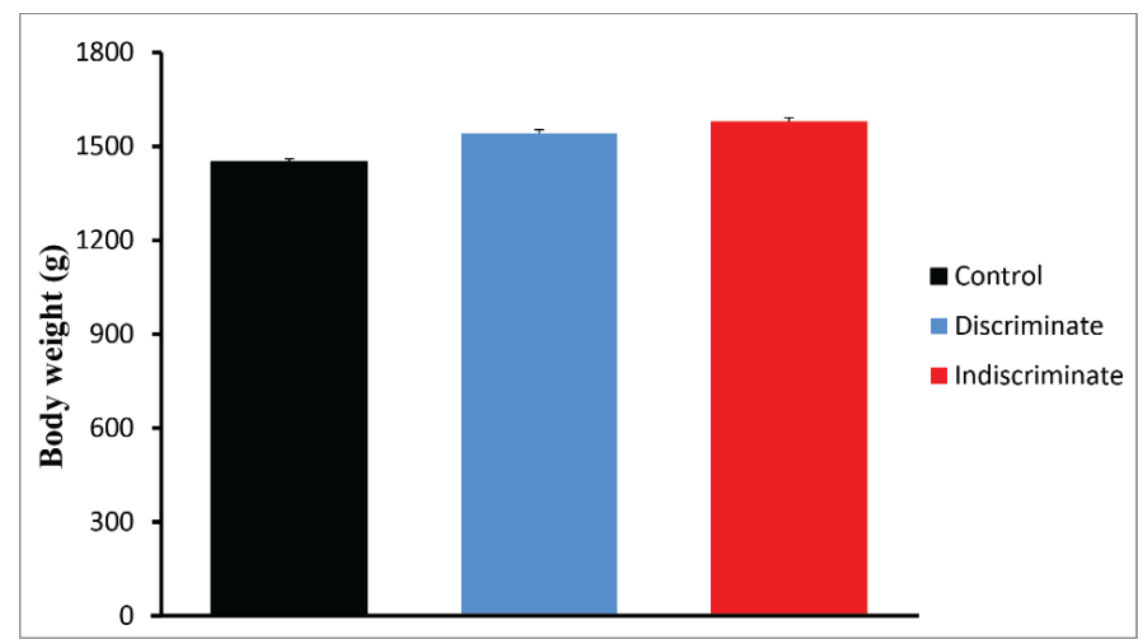

Figure 3. Body weight (gm) on $30^{\text {th }}$ day. 


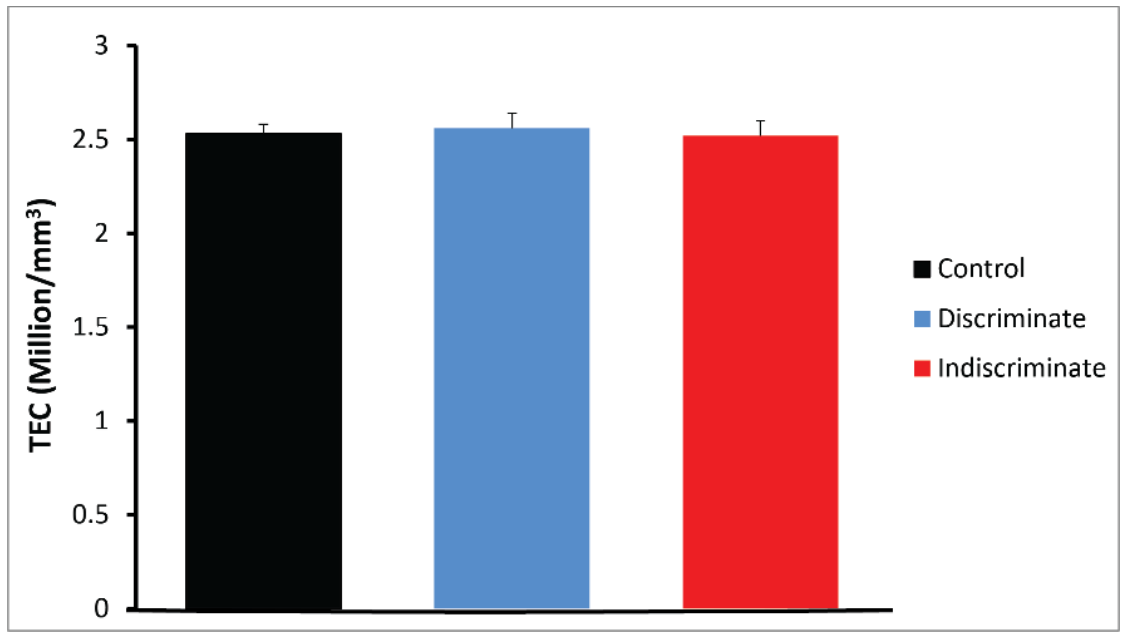

Figure 4. TEC (Million/ $\mathrm{mm}^{3}$ ) of three different groups.

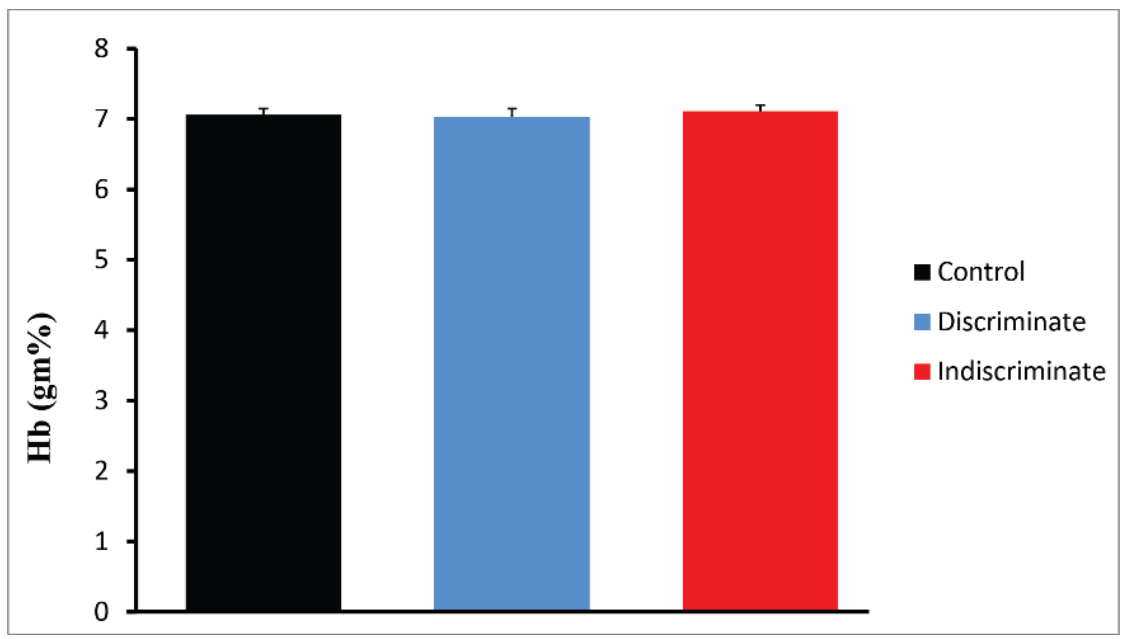

Figure 5. Hb (gm \%) of three different groups.

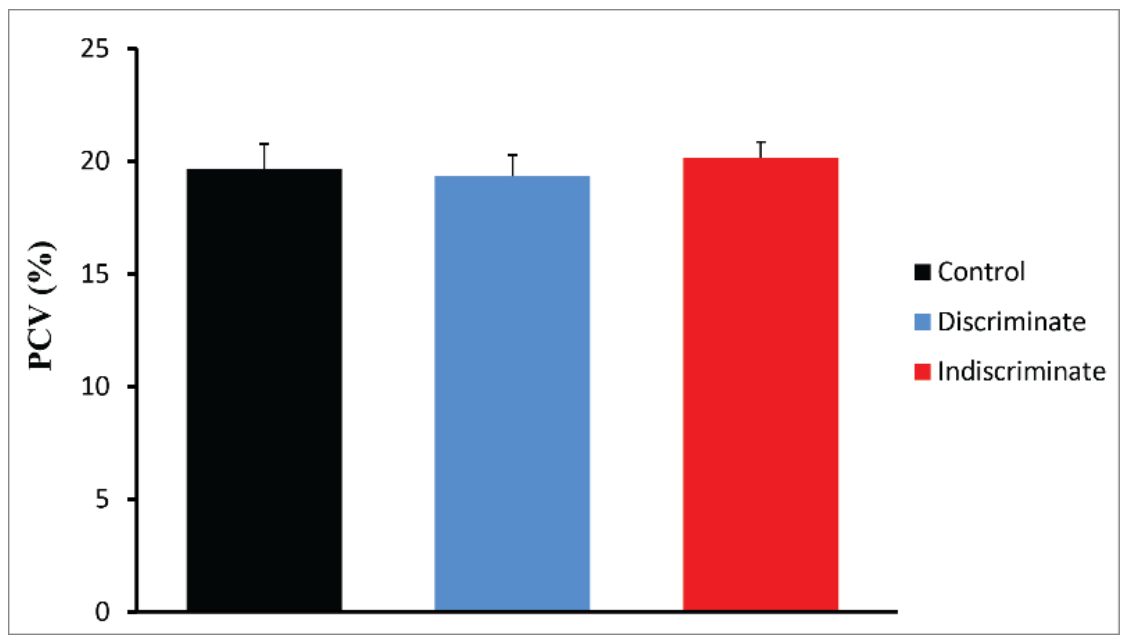

Figure 6. PCV (\%) of three different groups. 


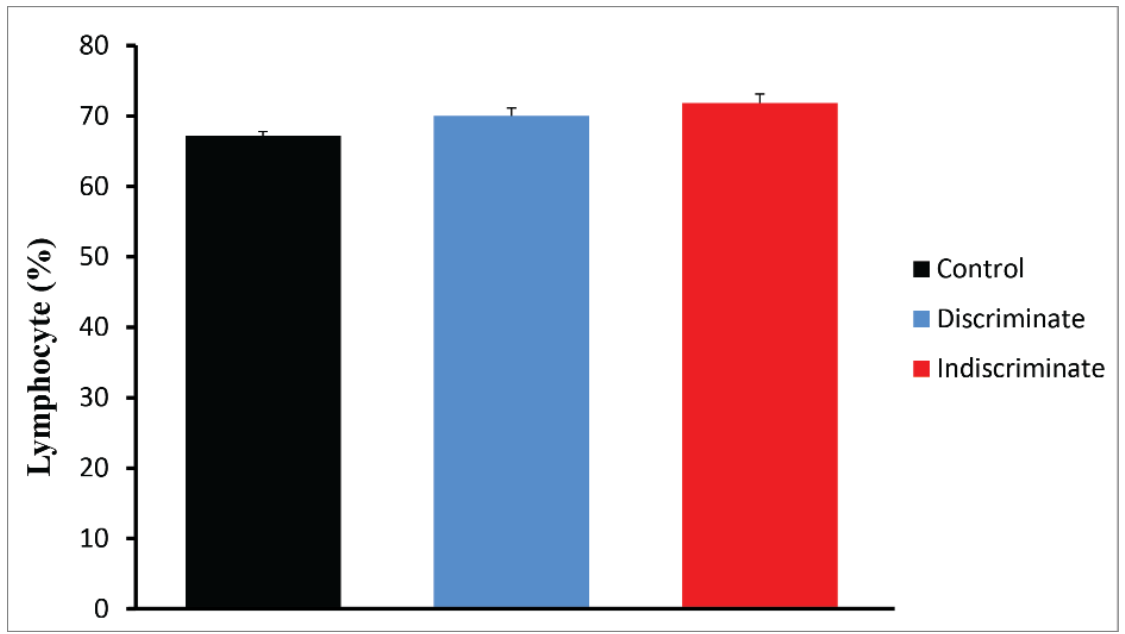

Figure 7. Lymphocyte (\%) count of three different groups.

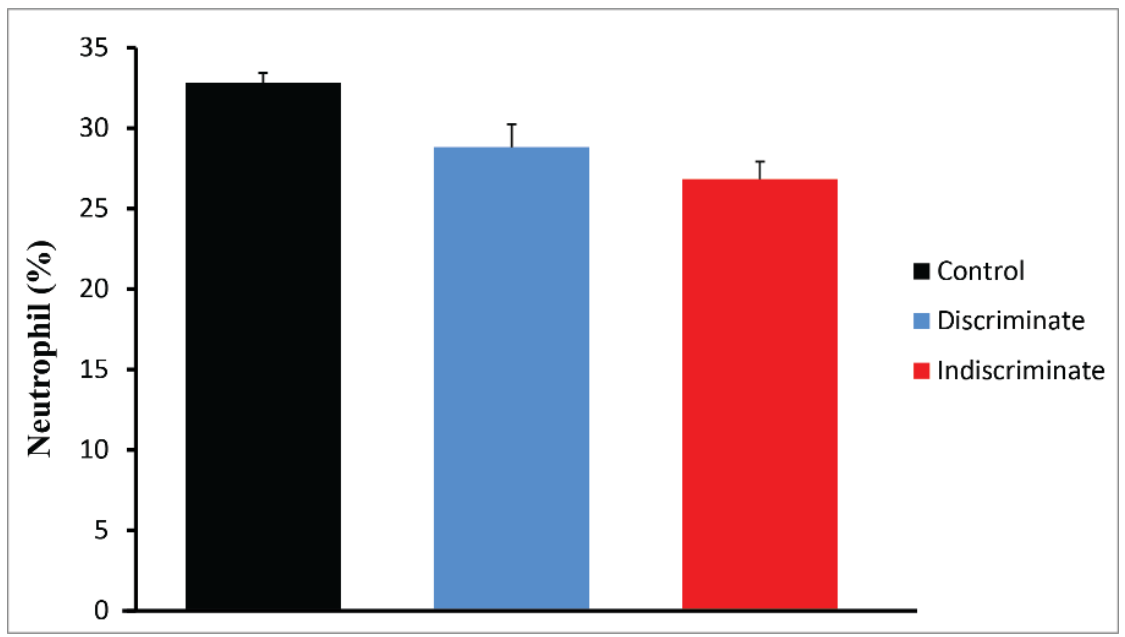

Figure 8. Neutrophil (\%) count of three different groups.

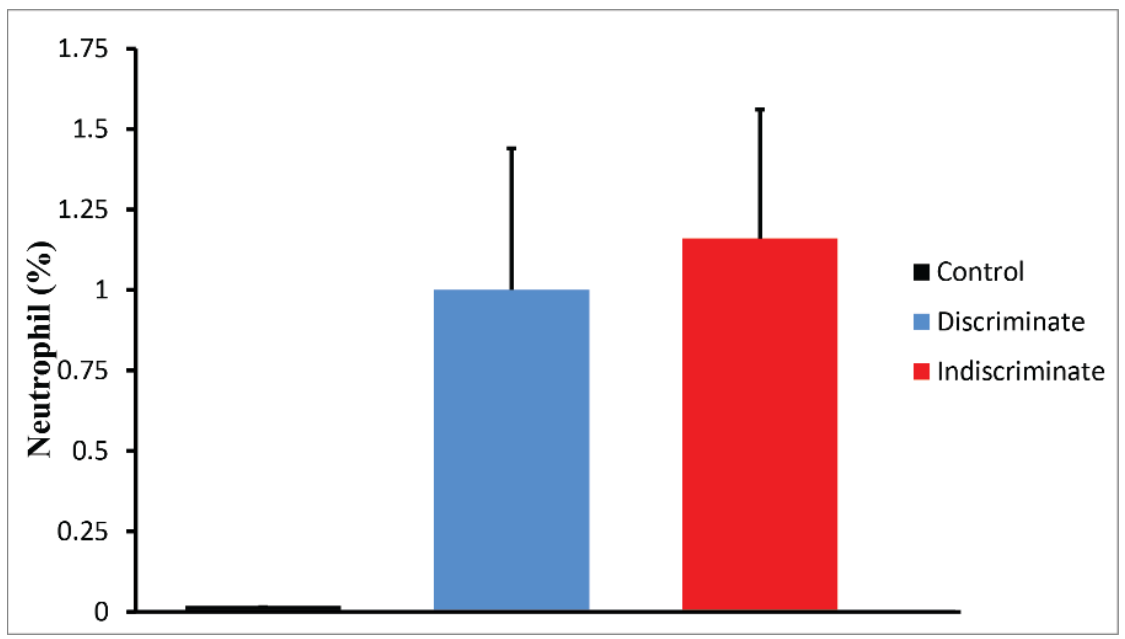

Figure 9. Eosinophil (\%) count of three different groups. 


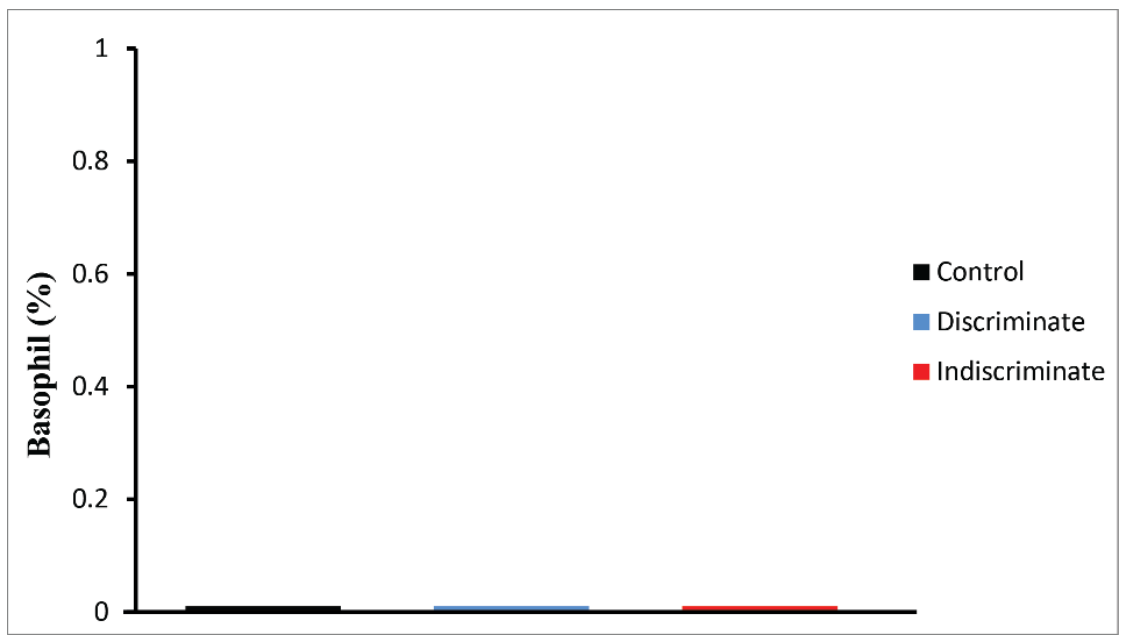

Figure 10. Basophil (\%) count of three different groups.

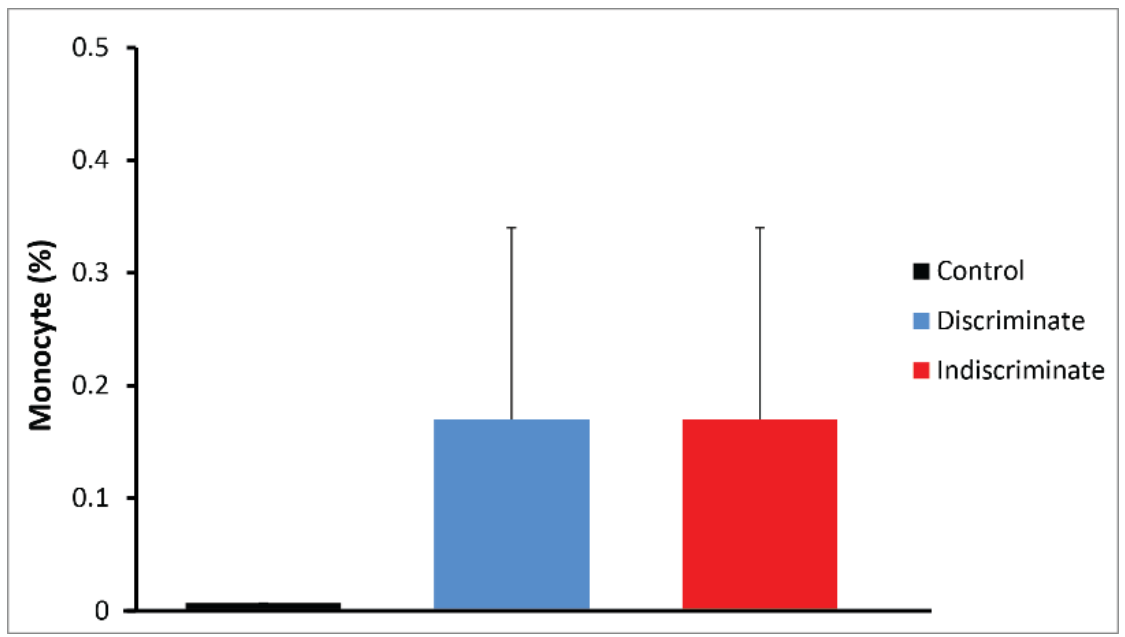

Figure 11. Monocyte (\%) count of three different groups.

\section{Discussion}

Poultry is one of the most widespread food industries worldwide. Chicken is the most commonly farmed species. Commercial production of poultry farming has been increasing day by day to fulfil the increasing food demand. In this production system antibiotics are used to enhance growth, feed efficiency and reduce diseases and thus consumers get poultry products such as meat and egg in reasonable cost (Salehzadeh et al., 2007). Although, using of antimicrobials is very beneficial but residues in produced products have negative impact on human health. Therefore, the aim of our study was to evaluate the effects of discriminate and indiscriminate use of colistin sulphate on body weight gain and haematological parameters in broiler chicken.

At the start of antibiotic administration $\left(16^{\text {th }}\right.$ day), there was no significant differences on body weight gain among three groups. But from $23^{\text {th }}$ day to the end of experiment ( $31^{\text {st }}$ day), the average body weight gain was the highest in indiscriminate antibiotic group which was $1408.22 \pm 133.49 \mathrm{gm}$ whereas, the lowest was $1243.81 \pm 173.19 \mathrm{gm}$ in control group. Our findings were validated by the similar finding of (Tabidi et al., 2013). Antibiotics has an ability to kill or inhibit the growth of bacteria, or microorganisms. Antibiotics continue to be used in the poultry industry as growth stimulants and therapeutic agents. The use of antibiotics, including chlortetracycline as growth promoters to increase production performance and to decrease mortality, was recommended to be banned by European Union (Perreten, 2003).

However, due to the fact that continued use of tends to stimulate development of resistance from harmful microorganisms, there is currently an outcry from the consumer society and health sector to band their use as feed additives in animal and poultry feeds (Cavazzoni et al., 1998; Jin et al., 1998; Yeo and Kim, 1997; Mohan et al., 1996) and residues in chicken meat products can be harmful to consumers. The control of infections and enhancement of live performance through a non-antibiotic approach is thus urgently required. 
(Al-Mayah et al., 2005) mentioned that broiler exposed to therapeutic and double doses of antibiotic based medical products have no significant impact on the haematological parameters of broilers.

The use of colistin sulphate for a specific period had some effects on haematological parameters. The highest mean values of total erythrocyte counts (Million $/ \mathrm{mm}^{3}$ ) were $2.56 \pm 0.08$ for the discriminate group, $2.53 \pm 0.05$ for the control group and $2.52 \pm 0.08$ for the indiscriminate group. Haemoglobin (gm\%) mean values were of $7.06 \pm 0.09$ for the control group, $7.03 \pm 0.12$ for the discriminate group and $7.13 \pm 0.09$ for the indiscriminate group. In addition, packed cell volume (\%) mean values were $19.66 \pm 1.11$ for the control group, $19.33 \pm 0.95$ for the discriminate group and $20.16 \pm 0.70$ for the indiscriminate group. Analysed data showed there was a tend to change TEC, $\mathrm{Hb}$ and PCV after discriminate and indiscriminate use of colistin sulphate in broiler poultry.

However, there is no significant difference among three groups. (Trinca et al., 2015) also showed there is no significant change of PCV in broilers those receiving overdose of antibiotics.

The antibiotics have effects on haematopoiesis and haemolysis but the effects are not significant in small duration. The effect is large when chronic exposure occurs. (Burke and Cunha, 2003) stated that various antibiotics exert diverse effects on different elements of blood, some agents produce anaemia or leukopenia and some may cause pancytopenia. In agreement with previous reports stated above present study data indicates that discriminate and indiscriminate use of colistin sulphate have no significant influence on haematological parameters in broiler chicken.

In that experiment, different leucocyte count (lymphocyte, neutrophil, eosinophil, basophil and monocyte) was also analysed. The highest mean lymphocyte $(\%)$ was obtained from indiscriminate group that was $71.83 \pm 1.30$. The mean lymphocyte (\%) of control and discriminate group were $67.16 \pm 0.6$ and $70 \pm 1.15$ respectively. Analysed data showed significant differences between the three groups $(P<0.05)$. The dose-dependent decrease might be attributed to the $\mathrm{AD}_{3} \mathrm{E}$ vitamin complex in the product that is known to induce lymphopenia as a side effect (Fiţ et al., 2012).

Neutrophil (\%) levels were $32.83 \pm 0.60$ in the control group, $28.83 \pm 1.42$ and $26.83 \pm 1.10$ in discriminate and indiscriminate group respectively. The ordinary one-way ANOVA showed that the differences among means of control versus discriminate and indiscriminate groups were statistically significant $(P<0.05)$. There was significant difference between discriminate and indiscriminate groups.

Eosinophil (\%) had a mean value of $1.16 \pm 0.40$ in the indiscriminate group and control and discriminate groups were $0 \pm 0$ and $1 \pm 0.44$. Eosinophil among the three different groups (Control, Discriminate and Indiscriminate) did not show any significant difference.

Basophil (\%) was rarely found on the blood smears, with values of 0.00 in all the investigated samples. This finding is similar to (Trinca et al., 2015).

Monocytes (\%) were at a mean level of $0.17 \pm 0.17 \%$ in both discriminate and indiscriminate groups, and control group were $0 \pm 0$ respectively. Monocyte among the three different groups (Control, Discriminate and Indiscriminate) did not show any significant difference.

Overall, the discussion, our experimental data shows that colistin sulphate treatment of haematological parameters particularly leucocyte cellular count such as lymphocyte, neutrophil, eosinophil, basophil and monocyte express the slightly influence the activity on lymphocyte and neutrophil. It indicates that antibiotic treatment influences the bone marrow but it is not significantly influence leucocyte profile of blood parameters in the short duration of treatment.

\section{Conclusions}

Antimicrobials are used in veterinary practices for animal and poultry production. The misuse of antimicrobials in food animal production has resulted in the emergence and dissemination of resistant pathogens and resistance genes. Antimicrobial resistance bacteria in food animals can affect not only animal health, but also public health when they enter into the food chain and environment leads to development of antibiotic resistant bacteria.

In our indoor trial mean body weight was highest for indiscriminate group and lowest in control group. Body weights of three groups of broilers showed no significant changes during the use of antibiotic.

In haematological parameters, total erythrocyte count, haemoglobin and packed cell volume did not show any significant changes among the three groups namely control, discriminate and indiscriminate groups respectively. In our experiment, both lymphocyte and neutrophil analysed data showed significant differences among the three groups (Control, Discriminate and Indiscriminate) whereas, eosinophil, basophil and monocyte did not show any significant difference. It is evident that the discriminate and indiscriminate use of colistin sulphate doesn't significantly influence the haematological parameters in the short duration of treatment and doesn't bear any fruitful effect on broiler. Further research is needed in this field, to ensure the effect of colistin sulphate antibiotic residues in the haematopoietic system and therefore the safety of their product. Both veterinarians and 
food producers have become extremely conscious of the need for high public confidence in the products they produce for human consumption.

\section{Acknowledgements}

This work was supported by the Ministry of Education, Government of the People's Republic of Bangladesh by a grant in research (Project No. 37.20.0000.004.033.020.2016.1053; LS2019925).

\section{Conflict of interest}

None to declare.

\section{Authors' contribution}

Saifa Nasar Trisha: research conduction, data analysis and article writing; Md. Shafiqul Islam: study, conception, design and data analysis; Md. Rakibul Hasan: manuscript preparation and data analysis; Md. Mahmudul Hasan Sikder: data interpretation and analysis; Most. Shumi Akhter Sathi: cooperation in research. All authors have read and approved the final manuscript.

\section{References}

Al-Mayah AS and JA AL-Ahmed, 2005. Influence of antibiotics treatment on haematological aspect in chickens. Int. J. Poult. Sci., 4: 323-325.

Bergen PJ, Li J, Rayner CR and Nation RL, 2006. Colistin methane sulfonate is an inactive prodrug of colistin against Pseudomonas aeruginosa. Antimicrob. Agents Chemother., 50: 1953-1958.

Bozorgmehri FMH, 2004. The effect colistin sulfate in feed on controlling of Salmonella enteritidis contamination in broiler farm. Archives of Razi Institute, 58: 105-110.

Bressan CR, A Kunz, W Schmidell and HM Soares, 2013. Toxicity of the colistin sulfate antibiotic used in animal farming to mixed cultures of nitrifying organisms. Water Air Soil Pollution, 224: 1441.

Burke A and MD Cunha, 2003. Principles of antibiotic formulary selection for Pandt committees. Medicinal Clinics of North America: 28. pp. 594-596.

Cavazzoni V, A Adami and C Castrovilli, 1998. Performance of broiler chickens supplemented with Bacillus coagulans as probiotic. Br. Poult. Sci., 39: 526-529.

Chung J and JL Wood, 1970. Oxidation of thiocyanate to cyanide catalyzed by hemoglobin. J. Biol. Chem., 246: 555-560.

Collell M and A Segura, 2013. Colistin a classical today essential in transition. Advances of Swine Technology, 97: 26-28.

Decolin D, P Leroy, A Nicolas and P Archimbault, 1997. Hyphenated liquid chromatographic method for the determination of colistin residues in bovine tissues. J. Chromatogr. Sci., 35: 557-564.

Donoghue DJ, 1993. Antibiotic residues in poultry tissues and eggs: human health concerns. Poult. Sci., 82: 618-621.

Elverdam I, P Larsen and E Lund, 1981. Isolation and characterization of three new polymyxins B and E by high-performance liquid chromatography. J. Chromatogr. A, 218: 653-61.

FAO, Food and Agriculture Organization of the United Nations 2006. Residue Evaluation of Certain Veterinary Drugs. 66th Meeting 2006. Rome, Italy. pp. 7-28.

Fiţ N, F Chirila, G Nadas, S Rapuntean, L Ognean, S Trinca and C Cuc Bouari, 2012. Haematological biochemical and microbiological studies at pigeons treated with a product based on metronidazole, oxytetracycline, furazolidone and bismuth sub-nitrate. Bulletin UASVM Veterinary Medicine, 69: 120-127.

Gilbert M, 2010. Detection and characterization of isolates of Escherichia coli of clinical and fecal origin in ponedroras hens. Theses of Doctoral. Madrid, Spain, pp. 1-184.

Jin LZ, YW Ho, N Abdullah, MA Ali and S Jalaludin, 1998. Effects of adherent Lactobacillus cultures on growth, weight of organs and intestinal microflora and volatile fatty acids in broilers. Anim. Feed Sci. Technol., 70: 197-209.

Khan AT and F Zafar 2005. Haematological study in response of varying doses of estrogen in broiler chicken. Int. J. Poult. Sci., 4: 748-751.

Koch Weser J, VW Sidel, EB Federman, P Kanarek, DC Finer and AE Eaton, 1970. Adverse effects of sodium colistimethate. Manifestations and specific reaction rates during 317 courses of therapy. Ann. Intern. Med., 72: 857-868.

Lamberg SL and R Rothstein, 1977. Laboratory Manual of Hematology and Urinalysis. Avi Publishing Company, Inc, Westport Connec-ticut, USA. Language book society (ELBS). 1st edition. 
Lee HJ, MH Lee and PD Ruy, 2001. Public health risks: chemical and antibiotic residues. J. Anim. Sci., 14: 402-413.

Mejia WJ, 2003. Epidemiology of swine salmonellosis on farms in catalonia and determination of risk factors for infection. Theses of Doctoral. Autonomous University of Barcelona, Spain, pp. 1-98.

Tabidi MH, AM Mukhtar and HI Mohammed, 2013. Effects of probiotic and antibiotic on performance and growth attributes of broiler chicks. Glob. J. Med. Plant Res., 1: 136-142.

Mohan B, R Kadirvel, A Natarajan and M Bhaskaran, 1996. Effect of probiotic supplementation on growth, nitrogen utilisation and serum cholesterol in broilers. Br. Poult. Sci., 37: 395-401.

Salehzadeh FA, N Salehzadeh, R Rokni, A Madani and F Golchinefar, 2007. Enrofloxacin residue in chicken tissues from Tehran slaughter houses in Iran. Pak. J. Nutr., 6: 409-413.

Singh S, S Shukla, N Tandia, N Kumar and R Paliwal, 2014. Antibiotic residues: a global challenge. Pharma Science Monitor, 5: 184-197.

Stolker AAM and UT Brinkman, 2005. Analytical strategies for residue analysis of veterinary drugs and growth promoting agents in food-producing animals - a review. J. Chromatogr. A, 1067: 15-53.

Tollefson L and MA Miller, 2011. Antibiotic use in food animals: controlling the human health impact. Journal of Food International, 83: 245-254.

Trinca SF, L Ognean, V Chiurciu, C Chiurciu, A Arion and I Dumitru, 2015. The influence of different doses of oxytetracycline on some haematological parameters in broilers. Bulletin UASVM Veterinary Medicine, $72(1)$.

Wallace SJ, J Li, RL Nation, CR Rayner, D Taylor, D Middleton, RW Milne, K Coulthard and JD Turnidge, 2008. Subacute toxicity of colistin methane sulphonate in rats: comparison of various intravenous dosage regimens. Antimicrob. Agents Chemother., 52: 1159-1161.

Wolinsky E and J D Hines, 1962. Neurotoxic and nephrotoxic effects of colistin in patients with renal disease. N. Engl. J. Med., 266: 759-768.

Xu Y, X Tian, C Ren, H Huang, X Zhang, X Gong, H Liu, Z Yu and L Zhang, 2012. Analysis of colistin A and $\mathrm{B}$ in fishery products by ultra-performance liquid chromatography with positive electrospray ionization tandem mass spectrometry. Journal of Chromatography B Analytical Technology Biomedical Life Science, 899: 14-20.

Yeo J and KI Kim, 1997. Effect of feeding diets containing an antibiotic, a probiotic, or yucca extract on growth and intestinal urease activity in broiler chicks. Poult. Sci., 76: 381-385. 